\title{
LARGE-TIME BEHAVIOR OF SOLUTIONS TO AN INITIAL-BOUNDARY VALUE PROBLEM ON THE HALF LINE FOR SCALAR VISCOUS CONSERVATION LAW*
}

\author{
ITSUKO HASHIMOTO ${ }^{\dagger}$ AND AKITAKA MATSUMURA ${ }^{\dagger}$
}

\begin{abstract}
We study the large-time behavior of the solution to an initial boundary value problem on the half line for scalar conservation law, where the data on the boundary and also at the far field are prescribed. In the case where the flux is convex and the corresponding Riemann problem for the hyperbolic part admits the transonic rarefaction wave (which means its characteristic speed changes the sign), it is known by the work of Liu-Matsumura-Nishihara ('98) that the solution tends toward a linear superposition of the stationary solution and the rarefaction wave of the hyperbolic part. In this paper, it is proved that even for a quite wide class of flux functions which are not necessarily convex, such the superposition of the stationary solution and the rarefaction wave is asymptotically stable, provided the rarefaction wave is weak. The proof is given by a technical $L^{2}$-weighted energy method.
\end{abstract}

Key words. large-time behavior, viscous conservation law, initial boundary value problem

AMS subject classifications. 35B40, 35L65, 35K20

1. Introduction. In this paper, we study the following initial-boundary value problem on the half line for scalar viscous conservation law:

$$
\begin{cases}u_{t}+f(u)_{x}=u_{x x}, & x>0, t>0, \\ u(0, t)=u_{-}, & t>0, \\ \lim _{x \rightarrow \infty} u(x, t)=u_{+}, & t>0, \\ u(x, 0)=u_{0}(x), & x>0,\end{cases}
$$

where the flux $f$ is a given $C^{2}$ function of $u$ satisfying $f(0)=f^{\prime}(0)=0, u_{ \pm}$ are given constants, and the initial data $u_{0}$ is assumed to satisfy $u_{0}(0)=u_{-}$and $\lim _{x \rightarrow \infty} u_{0}(x)=u_{+}$as the compatibility conditions.

We are interested in the large-time behavior of the solution which is determined by the shape of the flux $f(u)$ and the given constants $u_{ \pm}$. It is known that the asymptotic behavior is closely related to the solution of the corresponding Riemann problem for the hyperbolic part (c.f. [2], [3]):

$$
\left\{\begin{array}{l}
u_{t}+f(u)_{x}=0, \quad x \in R, t>0, \\
u(x, 0)= \begin{cases}u_{+}, & x>0 \\
u_{-}, & x<0\end{cases}
\end{array}\right.
$$

In the case where the flux $f$ is convex $\left(f^{\prime \prime}>0\right)$ and the Riemann Problem (1.2) has the rarefaction wave solution, Liu-Matsumura-Nishihara ('98 [2]) showed that depending on the signs of the characteristic speeds $f^{\prime}\left(u_{ \pm}\right)$, the large-time behavior of the solution is classified into the three cases:

(a) $f^{\prime}\left(u_{-}\right)<f^{\prime}\left(u_{+}\right) \leq 0, \quad$ (equivalently $\left.u_{-}<u_{+} \leq 0\right)$,

(b) $0 \leq f^{\prime}\left(u_{-}\right)<f^{\prime}\left(u_{+}\right), \quad$ (equivalently $0 \leq u_{-}<u_{+}$),

\footnotetext{
*Received June 21, 2007; accepted for publication November 14, 2007.

$\dagger$ Department of Pure and Applied Mathematics, Graduate School of Information Science and Technology, Osaka University, Toyonaka, Osaka 560-0043, Japan (itsuko@cas.cmc.osaka-u.ac.jp; akitaka@math.sci.osaka-u.ac.jp).
} 
(c) $f^{\prime}\left(u_{-}\right)<0<f^{\prime}\left(u_{+}\right), \quad$ (equivalently $\left.u_{-}<0<u_{+}\right)$,

where we again assume $f(0)=f^{\prime}(0)=0$ without loss of generality. More precisely, they showed the followings. In the case (a) where all the characteristic speeds of the rarefaction wave are non-positive, the solution of (1.1) eventually tends toward the stationary solution $\phi$ which connects $u_{-}$to $u_{+}$, where $\phi=\phi(x)$ is defined by the solution of the corresponding stationary problem to (1.1) :

$$
\left\{\begin{array}{l}
f(\phi)_{x}=\phi_{x x}, \quad x>0, \\
\phi(0)=u_{-}, \quad \phi(+\infty)=u_{+} .
\end{array}\right.
$$

In the case (b) where all the characteristic speeds of the rarefaction wave are nonnegative, the solution of (1.1) eventually tends toward the rarefaction wave $\psi^{R}$ itself which connects $u_{-}$to $u_{+}$, where $\psi^{R}=\psi^{R}\left(\frac{x}{t}\right)$ is concretely given by

$$
\psi^{R}\left(\frac{x}{t}\right)= \begin{cases}u_{-}, & x \leq f^{\prime}\left(u_{-}\right) t \\ \left(f^{\prime}\right)^{-1}\left(\frac{x}{t}\right), & f^{\prime}\left(u_{-}\right) t \leq x \leq f^{\prime}\left(u_{+}\right) t, \\ u_{+}, & x \geq f^{\prime}\left(u_{+}\right) t .\end{cases}
$$

In the case (c) where the rarefaction wave is transonic, that is, the characteristic speeds change the sign, the solution of (1.1) eventually tends toward the linear superposition of the stationary solution $\phi$ connecting $u_{-}$to 0 and the rarefaction wave $\psi^{R}$ connecting 0 to $u_{+}$.

Focusing on the most interesting case (c), $u_{-}<0<u_{+}$, we can naturally expect that the superposition $\phi+\psi^{R}$ is asymptotically stable for more general flux $f(u)$ which is convex for $u>0$ but not necessarily convex for $u<0$ as long as positive for $u \neq 0$, because even for such general flux the stationary solution $\phi$ connecting $u_{-}$to 0 is easily seen to exist. There have been only a few results on such cases without the convexity condition. Nagase [7] studied in her master thesis the case where the flux $f(u)$ satisfies

$$
\begin{aligned}
& f(0)=f^{\prime}(0)=0, \\
& \exists u_{*}<0 \text { s.t. } f\left(u_{*}\right)=0, \text { and } f(u)>0, u \in\left(u_{*}, \infty\right), u \neq 0, \\
& \exists \bar{u}_{*} \in\left(u_{*}, 0\right) \text { s.t. } f^{\prime \prime}\left(\bar{u}_{*}\right)=0, \\
& f^{\prime \prime \prime}(u)>0, \quad u \in R .
\end{aligned}
$$

Here, it is noted that the condition (1.5) implies $f^{\prime \prime}(u) \gtrless 0$ for $u \gtrless \bar{u}_{*}$, and $\exists \tilde{u}_{*} \in$ $\left(u_{*}, \bar{u}_{*}\right)$ s.t. $f^{\prime}\left(\tilde{u}_{*}\right)=0$. In this case, the superposition $\phi+\psi^{R}$ is expected to be asymptotically stable for $u_{-} \in\left(u_{*}, 0\right)$. A typical example which satisfies (1.5) is $f(u)=u^{2}\left(u-u_{*}\right)$, where $\bar{u}_{*}$ and $\tilde{u}_{*}$ are given by $u_{*} / 3$ and $2 u_{*} / 3$ respectively. Then she showed that for sufficiently small $\varepsilon>0$, if $u_{-} \in\left(\tilde{u}_{*}-\varepsilon, 0\right)$ and $0<u_{+}<\varepsilon$, the superposition $\phi+\psi^{R}$ is asymptotically stable. The proof is given by using a $L^{2}$-weighted energy method as in the previous works ([1], [3], [6]) where in order to show the asymptotic stability of viscous shock profile for non-convex state equations they manipulate a weight function constructed by viscous shock profile itself. She also used a suitable weight function constructed by the stationary solution $\phi$. However the case $u_{-} \in\left(u_{*}, \tilde{u}_{*}-\varepsilon\right)$ has been left open. In this paper, it is shown that we can solve this open question and even can make the condition (1.5) much weaker as

$$
\begin{aligned}
& f(0)=f^{\prime}(0)=0, \quad f^{\prime \prime}(0)>0, \\
& f(u)>0, \quad u \in\left[u_{-}, 0\right) .
\end{aligned}
$$


Under the condition (1.6), we show that if $u_{+}$is positive but sufficiently small, then the superposition $\phi+\psi^{R}$ is asymptotically stable. For the proof, we employ a technique in Matsumura-Mei [4] to obtain the a priori estimate of the solution, where they manipulate not only a weight function but also a transformation of the unknown functions in order to prove the asymptotic stability of viscous shock profile for a system of visco-elasticity with a non-convex nonlinearity.

This paper is organized as follows. We state the main theorem and explain the scheme of the proof in the Section 2. Making use of the linearized equation with $u_{+}=0$, we explain the essence how to construct our weight function in the Section 3 . We finally prove a priori estimate in the Section 4 , which completes the proof of the main theorem.

Some Notations. We denote by $C$ generic positive constants unless they need to be distinguished. For function spaces, $L^{2}=L^{2}((0, \infty))$ and $H^{k}=H^{k}((0, \infty))$ denote the usual Lebesgue space of square integrable functions and $k$-th order Sobolev space on the half line $(0, \infty)$ with norms $\|\cdot\|$ and $\|\cdot\|_{k}$, respectively. We also denote by $H_{0}^{1}=H_{0}^{1}((0, \infty))$ the space of functions $f \in H^{1}$ with $f(0)=0$, as a subspace of $H^{1}$.

2. Main theorem. In this section, we state the main theorem and the scheme of the proof. Let us rewrite our problem:

$$
\begin{cases}u_{t}+f(u)_{x}=u_{x x}, & x>0, t>0, \\ u(0, t)=u_{-}, & t>0, \\ \lim _{x \rightarrow \infty} u(x, t)=u_{+}, & t>0, \\ u(x, 0)=u_{0}(x), & x>0,\end{cases}
$$

where we assume $u_{-}<0<u_{+}$, and $f \in C^{2}$ satisfies the conditions

$$
\begin{aligned}
& f(0)=f^{\prime}(0)=0, \quad f^{\prime \prime}(0)>0, \\
& f(u)>0, \quad u \in\left[u_{-}, 0\right) .
\end{aligned}
$$

We also assume the initial data $u_{0}$ satisfies

$$
u_{0}-u_{+} \in H^{1}, \quad u_{0}(0)=u_{-} .
$$

Noting that the conditions $f \in C^{2}$ and $f^{\prime \prime}(0)>0$ imply the existence of positive constants $r$ and $\nu$ satisfying

$$
f^{\prime \prime}(u) \geq \nu>0, \quad|u| \leq r
$$

we further assume

$$
u_{-}<0<u_{+} \leq r .
$$

Under these assumptions, as stated in the Introduction, the asymptotic state of the solution of (2.1) is expected to be the linear superposition of the corresponding stationary solution $\phi$ connecting $u_{-}$to 0 and the rarefaction wave $\psi^{R}$ connecting 0 to $u_{+}$. We first recall the properties of the stationary solution $\phi$ which is given by the solution to the boundary value problem for the ordinary differential equation:

$$
\left\{\begin{array}{l}
f(\phi)_{x}=\phi_{x x}, \quad x>0, \\
\phi(0)=u_{-}, \quad \phi(+\infty)=0 .
\end{array}\right.
$$


If we integrate the equation of (2.6) once, it is easy to see (2.6) is equivalent to the problem :

$$
\left\{\begin{array}{l}
\phi_{x}=f(\phi), \quad x>0 \\
\phi(0)=u_{-}
\end{array}\right.
$$

Then we have the following lemma which is proved in the same way as in [2] and [3], so we omit the proof.

Lemma 1. Assume $u_{-}<0$ and (2.2). Then, the boundary value problem (2.6) which is equivalent to (2.7) has a unique solution $\phi \in C^{3}([0, \infty))$ satisfying

$$
\left\{\begin{array}{l}
u_{-}<\phi(x)<0 \text { and } \phi_{x}(x)>0, \quad x>0 \\
|\phi(x)| \leq C(1+x)^{-1}, \quad x \geq 0
\end{array}\right.
$$

Next, we recall the definition of the rarefaction wave $\psi^{R}$. We start with the rarefaction wave to the Riemann problem for the Burgers equation with the given end states $w_{ \pm}\left(w_{-}<w_{+}\right)$:

$$
\left\{\begin{array}{l}
w_{t}+\left(\frac{1}{2} w^{2}\right)_{x}=0, \quad x \in R, t>0 \\
w(x, 0)= \begin{cases}w_{+}, & x>0 \\
w_{-}, & x<0\end{cases}
\end{array}\right.
$$

We denote the solution of $(2.8)$ by $w^{R}\left(x / t ; w_{-}, w_{+}\right)$which is exactly given by

$$
w^{R}\left(\frac{x}{t} ; w_{-}, w_{+}\right)= \begin{cases}w_{-}, & x \leq w_{-} t \\ \frac{x}{t}, & w_{-} t \leq x \leq w_{+} t \\ w_{+}, & x \geq w_{+} t\end{cases}
$$

Since $f^{\prime \prime}(u)>0$ for $|u| \leq r$ by (2.4), the rarefaction wave $\psi^{R}$ connecting 0 to $u_{+}$for the Riemann problem

$$
\left\{\begin{array}{l}
u_{t}+f(u)_{x}=0, \quad x \in R, t>0, \\
u(x, 0)= \begin{cases}u_{+}, & x>0, \\
0, & x<0\end{cases}
\end{array}\right.
$$

is given by

$$
\begin{aligned}
\psi^{R}\left(\frac{x}{t}\right) & =\left(f^{\prime}\right)^{-1}\left(w^{R}\left(\frac{x}{t} ; 0, f^{\prime}\left(u_{+}\right)\right)\right. \\
& = \begin{cases}0, & x \leq 0 \\
\left(f^{\prime}\right)^{-1}\left(\frac{x}{t}\right), & 0 \leq x \leq f^{\prime}\left(u_{+}\right) t \\
u_{+}, & x \geq f^{\prime}\left(u_{+}\right) t\end{cases}
\end{aligned}
$$

Then we define the rarefaction wave for the initial boundary problem (2.1) by the restriction of $\psi^{R}$ on the half line $\left.\psi^{R}\left(\frac{x}{t}\right)\right|_{x>0}$, and write it again as $\psi^{R}$ without confusion. Now we are ready to state our main theorem. 
Theorem 1 (Main Theorem). Assume (2.2),(2.3), and (2.5). Then, there exist a positive constant $\varepsilon$ such that, if $u_{+} \leq \varepsilon$ and $\left\|u_{0}-\phi-\psi^{R}(\cdot)\right\|_{1} \leq \varepsilon$, then the initial boundary value problem (2.1) has a unique global solution in time $u$ satisfying

$$
\left\{\begin{array}{l}
u-u_{+} \in C\left([0, \infty) ; H^{1}\right), \\
u_{x} \in L^{2}\left(0, T ; H^{1}\right), \quad T>0
\end{array}\right.
$$

and the asymptotic behavior

$$
\lim _{t \rightarrow \infty} \sup _{x>0}\left|u(x, t)-\phi(x)-\psi^{R}\left(\frac{x}{t}\right)\right|=0 .
$$

In what follows, let us show the scheme how to prove the Main Theorem. As in the previous papers $([2,3,5,6,7])$, we first make a smooth approximation of the rarefaction wave $\psi^{R}$, because the non-smoothness of $\psi^{R}$ causes a trouble in the process of handling the second derivative of the solution. Following the arguments in [5], define a smooth approximation $w(x, t)$ of $w^{R}\left(\frac{x}{t}\right)$ by the solution of the Cauchy problem

$$
\left\{\begin{array}{l}
w_{t}+\left(\frac{1}{2} w^{2}\right)_{x}=0, \quad x \in R, t>0, \\
w(0, x)=f^{\prime}\left(u_{+}\right) \tanh x, \quad x \in R,
\end{array}\right.
$$

and a smooth approximation $\psi(x, t)$ of $\psi^{R}\left(\frac{x}{t}\right)$ by

$$
\psi(x, t)=\left(f^{\prime}\right)^{-1}(w(x, t)), \quad x \geq 0 .
$$

Then we have the next lemma which is proved in the same way as in [5].

LEMma 2. Assume (2.2) and $0<u_{+} \leq r$. Then we have the followings: 1) $\psi(x, t)$ is the smooth solution of the initial boundary value problem

$$
\left\{\begin{array}{l}
\psi_{t}+f(\psi)_{x}=0, \quad x>0, t>0, \\
\psi(t, 0)=0, \quad t>0 \\
\psi(0, x)=\left(f^{\prime}\right)^{-1}\left(f^{\prime}\left(u_{+}\right) \tanh x\right), \quad x>0 .
\end{array}\right.
$$

2) $0<\psi(x, t)<u_{+}$and $\psi_{x}(x, t)>0, \quad x>0, t>0$.

3) For $1 \leq p \leq \infty$, there exists a positive constant $C_{p}$ such that

$$
\begin{aligned}
& \left\|\psi_{x}(t)\right\|_{L^{p}} \leq C_{p} \min \left(u_{+}, u_{+}^{\frac{1}{p}}(1+t)^{-1+\frac{1}{p}}\right) \\
& \left\|\psi_{x x}(t)\right\|_{L^{p}} \leq C_{p} \min \left(u_{+},(1+t)^{-1}\right)
\end{aligned}
$$

4) $\lim _{t \rightarrow \infty} \sup _{x>0}\left|\psi(x, t)-\psi^{R}\left(\frac{x}{t}\right)\right|=0$.

Now if we put

$$
\Phi(x, t)=\phi(x)+\psi(x, t)
$$

as the expected asymptotic state, it follows from the definitions of $\phi$ and $\psi$ that $\Phi$ approximately satisfies the equation of (2.1) as

$$
\Phi_{t}+f(\Phi)_{x}-\Phi_{x x}=-F(\phi, \psi),
$$


where

$$
F(\phi, \psi)=-\left(f^{\prime}(\phi+\psi)-f^{\prime}(\phi)\right) \phi_{x}-\left(f^{\prime}(\phi+\psi)-f^{\prime}(\psi)\right) \psi_{x}+\psi_{x x} .
$$

Define the deviation $v$ of $u$ from $\Phi$ by

$$
v(x, t)=u(x, t)-\Phi(x, t) .
$$

Then the problem (2.1) is reformulated in terms of $v$ in the form

$$
\left\{\begin{array}{l}
v_{t}+\{f(\Phi+v)-f(\Phi)\}_{x}-v_{x x}=F(\phi, \psi), \quad x>0, t>0, \\
v(0, t)=0, \quad t>0, \\
v(x, 0)=v_{0}(x):=u_{0}(x)-\phi(x)-\psi(x, 0), \quad x>0,
\end{array}\right.
$$

where we can see $v_{0} \in H_{0}^{1}$ by the assumption (2.3). The theorem for the reformulated problem (2.20) we shall prove is

Theorem 2. Assume (2.2),(2.3), and (2.5). Then, there exists a positive constant $\varepsilon$ such that, if $\left\|v_{0}\right\|_{1} \leq \varepsilon$ and $0<u_{+} \leq \varepsilon$, then the initial boundary value problem (2.20) has a unique global solution in time $v$ satisfying

$$
\left\{\begin{array}{l}
v \in C\left([0, \infty) ; H_{0}^{1}\right) \\
v_{x} \in L^{2}\left(0, \infty ; H^{1}\right) \\
\lim _{t \rightarrow \infty} \sup _{x>0}|v(x, t)|=0 .
\end{array}\right.
$$

If we note

$$
\left\|v_{0}\right\|_{1}=\left\|u_{0}-\phi-\psi(\cdot, 0)\right\|_{1} \leq\left\|u_{0}-\phi-\psi^{R}(\cdot)\right\|_{1}+C\left|u_{+}\right|
$$

and particularly 4) of the lemma 1.4, the Main Theorem is a direct consequence of the Theorem 2. The Theorem 2 itself is proved by combining the local existence theorem together with the a priori estimate as in the previous papers. To state the local existence theorem precisely, we define the solution set for any interval $I \subset R$ and constant $M>0$ by

$$
X_{M}(I)=\left\{v \in C\left(I ; H_{0}^{1}\right) ; v_{x} \in L^{2}\left(0, T ; H^{1}\right), \sup _{t \in I}\|v(t)\|_{1} \leq M\right\},
$$

and also generalize the initial boundary value problem for any constant $\tau \geq 0$ as

$$
\left\{\begin{array}{l}
v_{t}+\{f(\Phi+v)-f(\Phi)\}_{x}-v_{x x}=F(\phi, \psi), \quad x>0, t>\tau, \\
v(0, t)=0, \quad t>\tau, \\
v(x, \tau)=v_{\tau}(x), \quad x>0, \quad v_{\tau} \in H_{0}^{1} .
\end{array}\right.
$$

Then we state the local existence theorem.

Proposition 1 (local existence). For any positive constant $M$, there exists a positive constant $t_{0}=t_{0}(M)$ which is independent of $\tau$ such that if $\left\|v_{\tau}\right\|_{1} \leq M$, the initial boundary value problem $(2.22)$ has a unique solution $v \in X_{2 M}\left(\left[\tau, \tau+t_{0}\right]\right)$.

It is noted that the problem (2.22) is reduced to the integral equation

$$
\begin{aligned}
v(x, t)= & \int_{0}^{\infty} G(x, y, t-\tau) v_{\tau}(y) d y+ \\
& +\int_{\tau}^{t} \int_{0}^{\infty} G(x, y ; t-s)\left(-(f(\phi+v)-f(\phi))_{x}+F(\phi, \psi)\right)(s) d y d s,
\end{aligned}
$$


where $G(x, y ; t)$ is the Green kernel of the Dirichlet zero boundary value problem for the linear heat equation on the half line, which is concretely given by

$$
G(x, y ; t)=\frac{1}{\sqrt{4 \pi t}}\left(e^{-\frac{(x-y)^{2}}{4 t}}-e^{-\frac{(x+y)^{2}}{4 t}}\right) .
$$

Since we can prove the Proposition 1 by a standard iterative method, we omit the proof of the Proposition 1. Next, let us state the a priori estimate which is essential in this paper.

Proposition 2 (a priori estimate). Under the condition (2.2), there exist positive constants $\varepsilon$ and $C$ such that if $0<u_{+}<\varepsilon$ and $v \in X_{\varepsilon}([0, T])$ is the solution of the problem (2.22) for some $T>0$, then it holds

$$
\begin{aligned}
\|v(t)\|_{1}^{2} & +\int_{0}^{t}\left(\left\|\sqrt{\Phi_{x}} v(s)\right\|^{2}+\left\|v_{x}(s)\right\|_{1}^{2}\right) d s \\
& \leq C\left(\left\|v_{0}\right\|_{1}^{2}+\left|u_{+}\right|^{\frac{1}{6}}\right), \quad t \in[0, T] .
\end{aligned}
$$

Here we should note $\Phi_{x}=\phi_{x}+\psi_{x}>0$ by the Lemmas 1 and 2. The proof of the Proposition 2 is given in the sections 3 and 4 . Once the Propositions 1 and 2 are proved, the Theorem 2 is proved in a standard way as in the previous works. In fact, combining the local existence and a priori estimate, we can first prove the global existence of the solution in time by choosing $\left\|v_{0}\right\|_{1}$ and $u_{+}$suitably small. Then we can see the estimate $(2.23)$ holds even for $t \in[0, \infty)$, that is,

$$
\sup _{t \geq 0}\|v(t)\|_{1}, \int_{0}^{\infty}\left\|v_{x}(t)\right\|_{1}^{2} d t<\infty .
$$

By using the equation and the estimate (2.23), we can also have (cf. (4.23),(4.26))

$$
\int_{0}^{\infty}\left|\frac{d}{d t}\left\|v_{x}(t)\right\|^{2}\right| d t<\infty
$$

which implies

$$
\lim _{t \rightarrow \infty}\left\|v_{x}(t)\right\|=0 .
$$

Using Sobolev's embedding lemma, the estimates (2.23) and (2.24), we can easily have

$$
\sup _{x>0}|v(x, t)| \leq\left.\sqrt{2}|| v(t)\right|^{\frac{1}{2}}\left\|v_{x}(t)\right\|^{\frac{1}{2}} \leq C\left\|v_{x}(t)\right\|^{\frac{1}{2}} \rightarrow 0, \quad t \rightarrow \infty,
$$

which shows the asymptotic behavior of the solution. Thus, we can show the proof of the Theorem 2 by the Propositions 1 and 2 .

3. Weight function. In this section, we explain how to make the weight function which plays an essential role in our technical $L^{2}$-energy method. For simplicity of explanation, noting that $v$ and $u_{+}$is sufficiently small in the a priori estimate, we take the linearized equation of the problem (2.22) with $u_{+}=0$ (accordingly, $\Phi(x, t)=\phi(x))$

$$
\left\{\begin{array}{l}
v_{t}+\left\{f^{\prime}(\phi) v\right\}_{x}-v_{x x}=0, \quad x>0, t>0 \\
v(0, t)=0, \quad t>0, \\
v(x, 0)=v_{0}(x), \quad x>0, \quad v_{0} \in H_{0}^{1}
\end{array}\right.
$$


Let $v \in C\left([0, T] ; H_{0}^{1}\right) \cap L^{2}\left(0, T ; H^{2}\right)$ be a solution of (3.1). The most typical way to have the $L^{2}$ estimate which does not depend on $T$ is one to multiply the equation of (3.1) by $v$ and integrate it with respect to $x$ over $(0, \infty)$. Then, the integration by parts gives

$$
\frac{d}{d t} \int_{0}^{\infty} \frac{1}{2} v^{2} d x+\frac{1}{2} \int_{0}^{\infty} f^{\prime \prime}(\phi) \phi_{x} v^{2} d x+\int_{0}^{\infty} v_{x}^{2} d x=0 .
$$

If we note $\phi_{x}>0$, the estimate of (3.2) works well in the case $f^{\prime \prime}>0$, but not in the case $f$ is not convex because $f^{\prime \prime}$ changes its sign. In order to overcome this difficulty, we try to apply a weighted energy method as in ([1], [3], [6]) where to show the asymptotic stability of viscous shock profile for non-convex state equations, a weight function $w$ is manipulated as a function of the viscous shock wave itself. Take a weight funtion $w(\phi)$ as a function of $\phi$, and multiply the equation of (3.1) by $v w$ and integrate it over $(0, \infty)$. Then, noting the relation $\phi_{x}=f(\phi)$, we have

$$
\begin{array}{r}
\frac{d}{d t} \int_{0}^{\infty} \frac{1}{2} w(\phi) v^{2} d x+\int_{0}^{\infty}\left(\frac{1}{2} f^{\prime \prime} w-f^{\prime} w^{\prime}-\frac{1}{2} f w^{\prime \prime}\right)(\phi) \phi_{x} v^{2} d x \\
+\int_{0}^{\infty} w(\phi) v_{x}^{2} d x=0 .
\end{array}
$$

Under the condition (1.5), Nagase [7] succeeded in making a positive and smooth weight function $w(u)$ for $u \in\left[u_{-}, 0\right], u_{-} \in\left(\tilde{u}_{*}-\varepsilon, 0\right)$ so that it holds in (3.3)

$$
\left(\frac{1}{2} f^{\prime \prime} w-\frac{1}{2} w^{\prime \prime} f-f^{\prime} w^{\prime}\right)(u)>0, \quad u \in\left[u_{-}, 0\right] .
$$

Roughly speaking, she constructed $w(u)$ as almost identically constant in the region $f^{\prime \prime}>0$, and $-f^{\prime}+$ constant in the remaining region, and then patch them up on the whole $\left[u_{-}, 0\right]$. In fact, even in the region $f^{\prime \prime}<0$, the term $-w^{\prime \prime} f / 2-f^{\prime} w^{\prime}(=$ $\left.f^{\prime \prime \prime} w / 2+f^{\prime} f^{\prime \prime}\right)$ in (3.4) is positive as long as $f^{\prime}<0$ and $f^{\prime \prime \prime}>0$, and so plays a nice role to control the negative term $f^{\prime \prime} w$ and show the positivity of (3.4), which is a basic technical idea in [7]. However this choice of weight function can not be easily extended to a region $f^{\prime \prime}<0$ and $f^{\prime}>0$ because the term $-f^{\prime} w^{\prime}\left(=f^{\prime} f^{\prime \prime}\right)$ becomes negative and so causes a problem, which is a main reason why the case $u_{-} \in\left(u_{*}, \tilde{u}_{*}-\varepsilon\right)$ has been left open. In order to overcome this difficulty, we employ a technique in MatsumuraMei [4] where they manipulated not only a weight function but also a transformation of the unknown functions in order to prove the asymptotic stability of viscous shock profile for a system of visco-elasticity with a non-convex nonlinearity. Following their technique, we introduce a new unknown function $\tilde{v}$ by

$$
v(x, t)=\chi(\phi(x)) \tilde{v}(x, t),
$$

where $\chi(u)$ is a positive and smooth function on $\left[u_{-}, 0\right]$. Substitute (3.5) into (3.1), then we have the equation of $\tilde{v}$ as in the form

$$
\tilde{v}_{t}+\frac{1}{\chi}\left(f^{\prime} \chi \tilde{v}\right)_{x}-\frac{1}{\chi}(\chi \tilde{v})_{x x}=0 .
$$

Multiply (3.6) by $w \tilde{v}$ and integrate it over $(0, \infty)$, then we have

$$
\begin{array}{r}
\frac{d}{d t} \int_{0}^{\infty} \frac{1}{2} w \tilde{v}^{2} d x+\int_{0}^{\infty}\left\{\left(\frac{1}{2} f^{\prime \prime} w-f^{\prime} w^{\prime}-\frac{1}{2} f w^{\prime \prime}\right)+\right. \\
\left.+\frac{\chi^{\prime}}{\chi^{2}}\left(\chi f^{\prime} w+\chi f w^{\prime}-\chi^{\prime} f w\right)\right\} \phi_{x} \tilde{v}^{2} d x+\int_{0}^{\infty} w \tilde{v}_{x}^{2} d x=0 .
\end{array}
$$


So we may choose positive functions $\chi(u)$ and $w(u)$ on $\left[u_{-}, 0\right]$ so that

$$
\left\{\frac{1}{2} f^{\prime \prime} w-\frac{1}{2} w^{\prime \prime} f-f^{\prime} w^{\prime}+\frac{\chi^{\prime}}{\chi^{2}}\left(\chi f^{\prime} w+\chi f w^{\prime}-\chi^{\prime} f w\right)\right\}(u)
$$

is positive on $\left[u_{-}, 0\right]$. Now let us choose $\chi=w$, then (3.8) becomes

$$
\frac{1}{2}\left(f^{\prime \prime} w-f w^{\prime \prime}\right)
$$

Hence it is enough to seek a positive weight function $w(u)$ which makes (3.9) positive on $\left[u_{-}, 0\right]$. Under the condition $(2.2)$, we choose the function $w(u)$ by

$$
w(u)=f(u)+\delta g(u), \quad u \in\left[u_{-}, r\right]
$$

where $\delta$ is a positive constant and

$$
g(u)=-u^{2 m}+r^{2 m}, \quad u \in\left[u_{-}, r\right], m \geq 1 .
$$

Here it is noted that the interval $\left[u_{-}, 0\right]$ is extended to $\left[u_{-}, r\right]$ to treat the case $u_{+}>0$, and that the constant $\delta$ and the integer $m$ are properly chosen later.

LEMMA 3 (weight function). Under the condition (2.2), if we take $\delta$ sufficiently small and $m$ sufficiently large, the functions $\frac{1}{2}\left(f^{\prime \prime}(u) w(u)-f(u) w^{\prime \prime}(u)\right)$ and $w(u)$ are positive for $u \in\left[u_{-}, r\right]$.

Proof. First it is easy by the condition (2.2) to see that there exists a positive constant $\nu$ such that $f^{\prime \prime}(u) \geq \nu$ for $|u| \leq r$, and $f(u) \geq \nu$ for $u \in\left[u_{-},-r\right]$. Substituting (3.10) into (3.9), we have

$$
\begin{aligned}
f^{\prime \prime} w-f w^{\prime \prime} & =f^{\prime \prime}(f+\delta g)-f(f+\delta g)^{\prime \prime} \\
& =\delta\left(f^{\prime \prime} g-f g^{\prime \prime}\right) .
\end{aligned}
$$

We divide the interval $\left[u_{-}, r\right]$ into $\left[u_{-},-r\right]$ and $[-r, r]$. For $u \in\left[u_{-},-r\right]$, substituting (3.11) into (3.12), we obtain

$$
\begin{aligned}
\delta\left(f^{\prime \prime} g-f g^{\prime \prime}\right) & =\delta\left(f^{\prime \prime}\left(-u^{2 m}+r^{2 m}\right)+2 m(2 m-1) f u^{2(m-1)}\right) \\
& =2 m(2 m-1) \delta u^{2(m-1)}\left\{\frac{-1+\left|\frac{r}{u}\right|}{2 m(2 m-1)} u^{2} f^{\prime \prime}+f\right\} .
\end{aligned}
$$

Because $f^{\prime \prime}$ is bounded, $|r / u| \leq 1$ and $f(u) \geq \nu$ for $u \in\left[u_{-},-r\right]$, we can choose $m$ sufficiently large so that

$$
\frac{-1+\left|\frac{r}{u}\right|}{2 m(2 m-1)} u^{2} f^{\prime \prime}(u)+f(u) \geq \frac{1}{2} \nu, \quad u \in\left[u_{-},-r\right]
$$

Therefore, (3.13) and (3.14) imply

$$
\delta\left(f^{\prime \prime} g-f g^{\prime \prime}\right) \geq m(2 m-1) \delta r^{2(m-1)} \nu>0, \quad u \in\left[u_{-},-r\right] .
$$

For $|u| \leq r$, we further divide the interval to $|u| \leq r / 2$ and $r / 2 \leq|u| \leq r$. For $|u| \leq r / 2$, since $f^{\prime \prime}>0, g>0$ and $f \geq 0, g^{\prime \prime} \leq 0$, it clearly holds

$$
\delta\left(f^{\prime \prime} g-f g^{\prime \prime}\right)>0, \quad|u| \leq \frac{r}{2} .
$$


On the other hand, for $r / 2 \leq|u| \leq r$, since $f^{\prime \prime}>0, g \geq 0$ and $f>0, g^{\prime \prime}<0$, it easily holds

$$
\delta\left(f^{\prime \prime} g-f g^{\prime \prime}\right)>0, \quad \frac{r}{2} \leq|u| \leq r .
$$

Thus, it follows from (3.15), (3.16) and (3.17) that

$$
\left(f^{\prime \prime} w-f w^{\prime \prime}\right)(u)=\delta\left(f^{\prime \prime} g-f g^{\prime \prime}\right)(u)>0, \quad u \in\left[u_{-}, r\right] .
$$

Next, we prove $w(u)=f(u)+\delta g(u)$ to be positive. To do that, we again divide the interval $\left[u_{-}, r\right]$ to $\left[u_{-},-r\right], r / 2 \leq|u| \leq r$ and $|u| \leq r / 2$. Noting $f \geq \nu$ and $g$ is bounded for $u \in\left[u_{-},-r\right]$, we can take $\delta$ sufficiently small so that

$$
w(u)=f(u)+\delta g(u) \geq \frac{\nu}{2}>0, \quad u \in\left[u_{-},-r\right] .
$$

Because $f>0, g \geq 0$ for $r / 2 \leq|u| \leq r$ and $f \geq 0, g>0$ for $|u| \leq r / 2$, we can easily have

$$
w(u)=f(u)+\delta g(u)>0, \quad|u| \leq r .
$$

Thus (3.18),(3.19) and (3.20) complete the proof of the Lemma 3.

Applying the Lemma 3 to (3.7) with $\chi=w$, we can obtain a basic $L^{2}$-estimate of $\tilde{v}$ which is equivalent to the estimate of $v$. In the next section, we establish the desired a priori estimate for the nonlinear problem by making use of the weight function $w(u)$ in the Lemma 3.

4. Proof of a priori estimate. In this section, we give the proof of the Proposition 2. First, put

$$
N(T)=\sup _{0 \leq t \leq T}\|v(t)\|_{1},
$$

and then we suppose $N(T) \leq 1$ throughout this section. Now, motivated by the argument in the Section 3, we introduce a new unknown function $\tilde{v}$ by

$$
v(x, t)=w(\Phi(t, x)) \tilde{v}(x, t),
$$

where $\Phi(t, x)=\phi(x)+\psi(t, x)$ and $w=f+\delta g$ is the weight function in the Lemma 3. Since the Lemmas 1 and 2 imply $\Phi(x, t) \in\left[u_{-}, r\right], x \geq 0, t \geq 0$, we note that $w(\Phi(x, t))$ is well defined as weight function by the Lemma 3 , that is, smooth and satisfies

$$
\nu \leq w(\Phi(x, t)) \leq C, \quad x \geq 0, t \geq 0
$$

for some positive constants $\nu$ and $C$. Substituting (4.1) into the equation of (2.20), we get

$$
(w(\Phi) \tilde{v})_{t}+(f(\Phi+w(\Phi) \tilde{v})-f(\Phi))_{x}-(w(\Phi) \tilde{v})_{x x}=F(\phi, \psi) .
$$

Multiplying $\tilde{v}$ by (4.3) and integrating it over $(0, \infty)$, we have

$$
\begin{aligned}
& \left(\frac{1}{2} \int_{0}^{\infty} w(\Phi) \tilde{v}^{2} d x\right)_{t}+\int_{0}^{\infty} \frac{1}{2} w^{\prime}(\Phi) \psi_{t} \tilde{v}^{2} d x \\
& \quad+\int_{0}^{\infty}-(f(\Phi+w(\Phi) \tilde{v})-f(\Phi)) \tilde{v}_{x} d x+\int_{0}^{\infty}(w(\Phi) \tilde{v})_{x} \tilde{v}_{x} d x=\int_{0}^{\infty} \tilde{v} F d x .
\end{aligned}
$$


By using the fact that

$$
\begin{array}{r}
-\left(\int_{0}^{\tilde{v}}(f(\Phi+w(\Phi) \eta)-f(\Phi)) d \eta\right)_{x}=-(f(\Phi+w(\Phi) \tilde{v})-f(\Phi)) \tilde{v}_{x} \\
-\int_{0}^{\tilde{v}}\left(f^{\prime}(\Phi+w(\Phi) \eta)\left(1+w^{\prime}(\Phi) \eta\right)-f^{\prime}(\Phi)\right) \Phi_{x} d \eta
\end{array}
$$

we rewrite the third term on the left hand side of (4.4) as

$$
\begin{aligned}
& \int_{0}^{\infty}-(f(\Phi+w(\Phi) \tilde{v})-f(\Phi)) \tilde{v}_{x} d x \\
& =\int_{0}^{\infty}\left(\int_{0}^{\tilde{v}}\left(f^{\prime}(\Phi+w(\Phi) \eta)-f^{\prime}(\Phi)\right) d \eta\right) \Phi_{x} d x \\
& \quad+\int_{0}^{\infty}\left(\int_{0}^{\tilde{v}} f^{\prime}(\Phi+w(\Phi) \eta) w^{\prime}(\Phi) \eta d \eta\right) \Phi_{x} d x \\
& =: I_{1}+I_{2} .
\end{aligned}
$$

We further rewrite $I_{1}$ and $I_{2}$ by the Taylor's formula as

$$
\begin{aligned}
I_{1} & =\int_{0}^{\infty}\left(\int_{0}^{\tilde{v}} f^{\prime \prime}(\Phi) w(\Phi) \eta+O\left(\eta^{2}\right) d \eta\right) \Phi_{x} d x \\
& =\int_{0}^{\infty} \frac{1}{2} f^{\prime \prime}(\Phi) w(\Phi) \tilde{v}^{2} \Phi_{x} d x+\int_{0}^{\infty} O(|\tilde{v}|) \tilde{v}^{2} \Phi_{x} d x
\end{aligned}
$$

and

$$
\begin{aligned}
I_{2} & =\int_{0}^{\infty}\left(\int_{0}^{\tilde{v}} f^{\prime}(\Phi) w^{\prime}(\Phi) \eta+O\left(\eta^{2}\right) d \eta\right) \Phi_{x} d x \\
& =\int_{0}^{\infty} \frac{1}{2} f^{\prime}(\Phi) w^{\prime}(\Phi) \tilde{v}^{2} \Phi_{x} d x+\int_{0}^{\infty} O(|\tilde{v}|) \tilde{v}^{2} \Phi_{x} d x
\end{aligned}
$$

Hence, substituting (4.6) and (4.7) into (4.5), we have

$$
\begin{aligned}
& \int_{0}^{\infty}-(f(\Phi+w(\Phi) \tilde{v})-f(\Phi)) \tilde{v}_{x} d x \\
&=\int_{0}^{\infty} \frac{1}{2}\left(f^{\prime \prime} w+f^{\prime} w^{\prime}\right)(\Phi) \tilde{v}^{2} \Phi_{x} d x+\int_{0}^{\infty} O(|\tilde{v}|) \tilde{v}^{2} \Phi_{x} d x .
\end{aligned}
$$

We also rewrite the fourth term on the left hand side of (4.4) as

$$
\begin{aligned}
& \int_{0}^{\infty}(w(\Phi) \tilde{v})_{x} \tilde{v}_{x} d x \\
& =\int_{0}^{\infty}\left(w \tilde{v}_{x}^{2}+w^{\prime} \Phi_{x} \tilde{v} \tilde{v}_{x}\right) d x \\
& =\int_{0}^{\infty}\left(w \tilde{v}_{x}^{2}-\frac{1}{2} w^{\prime} \Phi_{x x} \tilde{v}^{2}-\frac{1}{2} w^{\prime \prime} \Phi_{x}^{2} \tilde{v}^{2}\right) d x \\
& =: \int_{0}^{\infty} w \tilde{v}_{x}^{2} d x+I_{3}+I_{4} .
\end{aligned}
$$


Now, recalling the relation $\Phi_{t}+f(\Phi)_{x}-\Phi_{x x}=-F$, we further rewrite $I_{3}$ as

$$
\begin{aligned}
I_{3} & =\int_{0}^{\infty}-\frac{1}{2} w^{\prime} \Phi_{x x} \tilde{v}^{2} d x \\
& =\int_{0}^{\infty} \frac{1}{2} w^{\prime}\left(-\Phi_{t}-f^{\prime}(\Phi) \Phi_{x}-F\right) \tilde{v}^{2} d x \\
& =\int_{0}^{\infty}\left(-\frac{1}{2} w^{\prime} f^{\prime}(\Phi) \Phi_{x} \tilde{v}^{2}-\frac{1}{2} w^{\prime} \psi_{t} \tilde{v}^{2}-\frac{1}{2} w^{\prime} F \tilde{v}^{2}\right) d x
\end{aligned}
$$

and $I_{4}$ as

$$
\begin{aligned}
I_{4} & =\int_{0}^{\infty}-\frac{1}{2} w^{\prime \prime} \Phi_{x}^{2} \tilde{v}^{2} d x \\
& =\int_{0}^{\infty}-\frac{1}{2} w^{\prime \prime}\left(\phi_{x}+\psi_{x}\right) \Phi_{x} \tilde{v}^{2} d x \\
& =\int_{0}^{\infty}-\frac{1}{2} w^{\prime \prime}\left(f(\Phi)+\phi_{x}+\psi_{x}-f(\phi+\psi)\right) \Phi_{x} \tilde{v}^{2} d x \\
& =\int_{0}^{\infty}-\frac{1}{2} w^{\prime \prime} f(\Phi) \Phi_{x} \tilde{v}^{2}-\frac{1}{2} w^{\prime \prime}\left(f(\phi)-f(\phi+\psi)+\psi_{x}\right) \Phi_{x} \tilde{v}^{2} d x \\
& =\int_{0}^{\infty}-\frac{1}{2} w^{\prime \prime} f(\Phi) \Phi_{x} \tilde{v}^{2} d x+\int_{0}^{\infty} O\left(|\psi|+\left|\psi_{x}\right|\right) \Phi_{x} \tilde{v}^{2} d x .
\end{aligned}
$$

Substituting (4.10) and (4.11) into (4.9), we have

$$
\begin{aligned}
\int_{0}^{\infty}(w(\Phi) \tilde{v})_{x} \tilde{v}_{x} d x \\
=\int_{0}^{\infty}-\frac{1}{2}\left(w^{\prime \prime} f+w^{\prime} f^{\prime}\right)(\Phi) \Phi_{x} \tilde{v}^{2} d x+\int_{0}^{\infty} O\left(|\psi|+\left|\psi_{x}\right|\right) \Phi_{x} \tilde{v}^{2} d x \\
\quad+\int_{0}^{\infty}\left(-\frac{1}{2} w^{\prime} \psi_{t} \tilde{v}^{2}+w \tilde{v}_{x}^{2}-\frac{1}{2} w^{\prime} F \tilde{v}^{2}\right) d x
\end{aligned}
$$

Thus, by (4.8) and (4.12), (4.4) reads

$$
\begin{aligned}
& \left(\frac{1}{2} \int_{0}^{\infty} w(\Phi) \tilde{v}^{2} d x\right)_{t}+\int_{0}^{\infty} \frac{1}{2}\left(w f^{\prime \prime}-w^{\prime \prime} f\right)(\Phi) \Phi_{x} \tilde{v}^{2} d x+\int_{0}^{\infty} w \tilde{v}_{x}^{2} d x \\
& =\int_{0}^{\infty}\left(\tilde{v} F+\frac{1}{2} w^{\prime} F \tilde{v}^{2}\right) d x+\int_{0}^{\infty} O\left(|\tilde{v}|+|\psi|+\left|\psi_{x}\right|\right) \Phi_{x} \tilde{v}^{2} d x
\end{aligned}
$$

Noting that the Sobolev's embedding lemma and Lemma 2 easily imply

$$
\int_{0}^{\infty} O\left(\tilde{v}+|\psi|+\left|\psi_{x}\right|\right) \Phi_{x} \tilde{v}^{2} d x \leq C\left(N(T)+\left|u_{+}\right|\right) \int_{0}^{\infty} \Phi_{x} \tilde{v}^{2} d x
$$

and also the Sobolev's embedding lemma and Young's inequality imply

$$
\begin{aligned}
\left|\int_{0}^{\infty}\left(\tilde{v} F+\frac{1}{2} w^{\prime} F \tilde{v}^{2}\right) d x\right| & \leq C \int_{0}^{\infty}|\tilde{v} \| F| d x \\
& \leq C\|\tilde{v}\|^{\frac{1}{2}}\left\|\tilde{v}_{x}\right\|^{\frac{1}{2}}\|F\|_{L^{1}} \\
& \leq \frac{1}{2}\left\|\sqrt{w} \tilde{v}_{x}\right\|^{2}+C\|F\|_{L^{1}}^{\frac{4}{3}},
\end{aligned}
$$


we can estimate (4.13) due to the Lemma 3 as

$$
\begin{aligned}
& \left(\frac{1}{2} \int_{0}^{\infty} w(\Phi) \tilde{v}^{2} d x\right)_{t}+\nu \int_{0}^{\infty} \Phi_{x} \tilde{v}^{2} d x+\frac{1}{2} \int_{0}^{\infty} w(\Phi) \tilde{v}_{x}^{2} d x \\
& \leq C\left(N(T)+\left|u_{+}\right|\right) \int_{0}^{\infty} \Phi_{x} \tilde{v}^{2} d x+C|| F \|_{L^{1}}^{\frac{4}{3}}
\end{aligned}
$$

for a positive constant $\nu$. Therefore, taking $N(T)+\left|u_{+}\right|$suitably small, we have

$$
\begin{aligned}
& \left(\frac{1}{2} \int_{0}^{\infty} w(\Phi) \tilde{v}^{2} d x\right)_{t}+\frac{\nu}{2} \int_{0}^{\infty} \Phi_{x} \tilde{v}^{2} d x+\frac{1}{2} \int_{0}^{\infty} w(\Phi) \tilde{v}_{x}^{2} d x \\
& \leq C\|F\|_{L^{1}}^{\frac{4}{3}} .
\end{aligned}
$$

Using the positivity of $w(4.2)$ and the fact

$$
\left\|v_{x}\right\|^{2}=\left\|(w \tilde{v})_{x}\right\|^{2}=\left\|w_{x} \tilde{v}+w \tilde{v}_{x}\right\|^{2} \leq C\left(\left\|\sqrt{\Phi_{x}} \tilde{v}\right\|^{2}+\left\|\tilde{v}_{x}\right\|^{2}\right),
$$

and integrating (4.17) with respect to $t$ over $(0, t)$, we have

$$
\begin{aligned}
\|v(t)\|^{2}+\int_{0}^{t}\left(\left\|\sqrt{\Phi_{x}} v(\tau)\right\|^{2}\right. & \left.+\left\|v_{x}(\tau)\right\|^{2}\right) d \tau \\
& \leq C\left(\left\|v_{0}\right\|^{2}+\int_{0}^{t}\|F(\tau)\|_{L^{1}}^{\frac{4}{3}} d \tau\right) .
\end{aligned}
$$

Next, we proceed to the estimate of $v_{x}$. Multiplying $-v_{x x}$ by the equation of (2.20) and integrating it with respect to $x$ over $(0, \infty)$, we have

$$
\begin{aligned}
\left(\frac{1}{2} \int_{0}^{\infty} v_{x}^{2} d x\right)_{t} & +\int_{0}^{\infty} v_{x x}^{2} d x=-\int_{0}^{\infty} F v_{x x} d x \\
& +\int_{0}^{\infty}\left(f^{\prime}(\Phi+v)\left(\Phi_{x}+v_{x}\right)-f^{\prime}(\Phi) \Phi_{x}\right) v_{x x} d x .
\end{aligned}
$$

We estimate the right hand side of (4.20) as

$$
\left|\int_{0}^{\infty} F v_{x x} d x\right| \leq \frac{1}{4}\left\|v_{x x}\right\|^{2}+C|| F \|^{2}
$$

and

$$
\begin{aligned}
\mid \int_{0}^{\infty}\left(f^{\prime}(\Phi\right. & \left.+v)\left(\Phi_{x}+v_{x}\right)-f^{\prime}(\Phi) \Phi_{x}\right) v_{x x} d x \mid \\
& \leq \int_{0}^{\infty} C\left(|v| \Phi_{x}+\left|v_{x}\right|\right)\left|v_{x x}\right| d x \\
& \leq \frac{1}{4}\left\|v_{x x}\right\|^{2}+C\left(\left\|\sqrt{\Phi_{x}} v\right\|^{2}+\left\|v_{x}\right\|^{2}\right) .
\end{aligned}
$$

Substituting (4.21) and (4.22) into (4.20), we have

$$
\begin{aligned}
\left(\frac{1}{2} \int_{0}^{\infty} v_{x}^{2} d x\right)_{t} & +\frac{1}{2} \int_{0}^{\infty} v_{x x}^{2} d x \\
& \leq C\left(\|F\|^{2}+\left\|\sqrt{\Phi_{x}} v\right\|^{2}+\left\|v_{x}\right\|^{2}\right) .
\end{aligned}
$$


Integrating (4.23) with respect to $t$ over $(0, t)$ and combining it with the estimate (4.19), we obtain

$$
\left\|v_{x}\right\|^{2}+\int_{0}^{t}\left\|v_{x x}\right\|^{2} d \tau \leq C\left(\left\|v_{0}\right\|_{1}^{2}+\int_{0}^{t}\left(\|F\|_{L^{1}}^{\frac{4}{3}}+\|F\|^{2}\right) d \tau\right) .
$$

Thus by (4.19) and (4.24), we have

$$
\begin{aligned}
\|v(t)\|_{1}^{2} & +\int_{0}^{t}\left(\left\|\sqrt{\Phi_{x}} v(\tau)\right\|^{2}+\left\|v_{x}(\tau)\right\|_{1}^{2}\right) d \tau \\
& \leq C\left(\left\|v_{0}\right\|_{1}^{2}+\int_{0}^{t}\|F(\tau)\|_{L^{1}}^{\frac{4}{3}}+\|F(\tau)\|^{2} d \tau\right) .
\end{aligned}
$$

Following the arguments in [2], we finally estimate the right hand side of (4.25) by using the Lemmas 1 and 2, and eventually can show

$$
\begin{aligned}
\|F(t)\|_{L^{1}}^{\frac{4}{3}} & \leq C\left|u_{+}\right|^{\frac{1}{6}}(1+t)^{-\frac{7}{6}} \log ^{\frac{4}{3}}(2+t), \\
\|F(t)\|^{2} & \leq C\left|u_{+}\right|^{\frac{1}{2}}(1+t)^{-\frac{3}{2}}
\end{aligned}
$$

Noting

$$
|F(\Phi)| \leq C\left(\left|\psi \phi_{x}\right|+\left|\psi_{x} \phi\right|+\left|\psi_{x x}\right|\right)
$$

we only show the estimates of $\left\|\psi \phi_{x}\right\|_{L^{1}}$ and $\left\|\psi \phi_{x}\right\|^{2}$ because the other terms can be obtained in the same way. Using the fact that the Lemma 1 implies

$$
\left|\phi_{x}(x)\right| \leq \frac{C}{(1+x)^{2}}, \quad x>0
$$

and the decay estimates of $\psi$ in the Lemma 2, we have

$$
\begin{aligned}
\left\|\psi \phi_{x}\right\|_{L^{1}} & \leq C \int_{0}^{\infty} \frac{\psi}{(1+x)^{2}} d x \\
& \leq C \int_{0}^{t} \frac{\psi}{(1+x)^{2}} d x+C \int_{t}^{\infty} \frac{\psi}{(1+x)^{2}} d x \\
& \leq C\left(\left[-\frac{\psi}{(1+x)}\right]_{0}^{t}+\int_{0}^{t} \frac{\psi_{x}}{(1+x)} d x\right)+C\|\psi\|_{L^{\infty}} \int_{t}^{\infty} \frac{1}{(1+x)^{2}} d x \\
& \leq C\left\|\psi_{x}\right\|_{L^{\infty}} \log (1+t)+C\left|u_{+}\right|(1+t)^{-1} \\
& \leq C\left\|\psi_{x}\right\|_{L^{\infty}}^{\frac{1}{8}}\left\|\psi_{x}\right\|_{L^{\infty}}^{\frac{7}{8}} \log (2+t)+C\left|u_{+}\right|^{\frac{1}{8}}\left|u_{+}\right|^{\frac{7}{8}}(1+t)^{-1} \\
& \leq C\left|u_{+}\right|^{\frac{1}{8}}(1+t)^{-\frac{7}{8}} \log (2+t)
\end{aligned}
$$




$$
\begin{aligned}
\left\|\psi \phi_{x}\right\|^{2} & \leq C \int_{0}^{\infty} \frac{\psi^{2}}{(1+x)^{4}} d x \\
& \leq C \int_{0}^{t} \frac{\psi^{2}}{(1+x)^{4}} d x+C \int_{t}^{\infty} \frac{\psi^{2}}{(1+x)^{4}} d x \\
& \leq C\left(\left[-\frac{\psi^{2}}{3(1+x)^{3}}\right]_{0}^{t}+\int_{0}^{t} \frac{\psi \psi_{x}}{3(1+x)^{3}} d x\right)+C\left|u_{+}\right|^{2}(1+t)^{-3} \\
& \leq C|| \psi_{x} \|_{L^{\infty}} \int_{0}^{t} \frac{\psi}{(1+x)^{3}} d x+C\left|u_{+}\right|^{2}(1+t)^{-3} \\
& \leq C|| \psi_{x} \|_{L^{\infty}}^{2} \int_{0}^{t} \frac{1}{(1+x)^{2}} d x+C\left|u_{+}\right|^{2}(1+t)^{-3} \\
& \leq C\left|u_{+}\right|^{\frac{1}{2}}(1+t)^{-\frac{3}{2}}
\end{aligned}
$$

and similarly

$$
\begin{aligned}
\left\|\psi_{x} \phi\right\|_{L^{1}}+\left\|\psi_{x x}\right\|_{L^{1}} & \leq C\left|u_{+}\right|^{\frac{1}{8}}(1+t)^{-\frac{7}{8}} \log (2+t), \\
\left\|\psi_{x} \phi\right\|^{2}+\left\|\psi_{x x}\right\|^{2} & \leq C\left|u_{+}\right|^{\frac{1}{2}}(1+t)^{-\frac{3}{2}}
\end{aligned}
$$

Hence (4.29), (4.30) and (4.31) prove the estimate (4.26). Then substituting (4.26) into (4.25), we finally have the desired a priori estimate for suitably small $N(T)+\left|u_{+}\right|$

$$
\begin{aligned}
\|v(t)\|_{1}^{2}+\int_{0}^{t}\left(\left\|\sqrt{\Phi_{x}} v(\tau)\right\|^{2}\right. & \left.+\left\|v_{x}^{2}(\tau)\right\|_{1}\right) d \tau \\
& \leq C\left(\left\|v_{0}\right\|_{1}^{2}+\left|u_{+}\right|^{\frac{1}{6}}\right), \quad t \in[0, T] .
\end{aligned}
$$

Thus the proof of the Proposition 2 is completed.

Acknowledgment. One of the authors heartily thanks to Dr. Chifune Kai for his constant encouragement and kind advices. This research is partially supported by the Grant-in-Aid for Scientific Research (B) (No. 19340037) of Japan Society for the Promotion of Science.

\section{REFERENCES}

[1] S. Kawashima And A. Matsumura, Stability of shock profiles in viscoelasticity with non-convex constitutive relations, Commun. Pure Appl. Math., 47 (1994), pp. 1547-1569.

[2] T.-P. Liu, A. Matsumura And K. Nishinara, Behaviors of solutions for the Burgers equation with boundary corresponding to rarefaction waves, SIAM J. Math. Anal., 29 (1998), pp. 293-308.

[3] T.-P. Liu And K. Nishinara, Asymptotic behavior for scalar viscous conservation laws with boundary effect, J. Differential Equations, 133 (1997), pp. 296-320.

[4] A. Matsumura and M. MeI, Nonlinear stability of viscous shock profile for a non-convex system of viscoelasticity, Osaka J. Math., 34 (1997), pp. 589-603.

[5] A. Matsumura and K. Nishinara, Asymptotic toward the rarefaction wave of solutions of a one-dimensional model system for compressible viscous gas, Japan J. Appl. Math., 3 (1986), pp. 1-13.

[6] - Asymptotic stability of traveling waves for scalar viscous conservation laws with non-convex nonlinearity, Commun. Math. Phys., 165 (1994), pp. 83-96.

[7] J. NAGASE, Large time behavior of solutions to an initial-boundary value problem on the half space for the generalized Burgers equation, Master thesis of Department of Mathematics, Guraduate School of Science, Osaka University, 2000. 
I. HASHIMOTO AND A. MATSUMURA 